\title{
A new name for the Neotropical genus Aniarella Enderlein (Diptera, Sciaridae)
}

\author{
Hüseyin Özdikmen ${ }^{1} \&$ Kadir Başar ${ }^{1}$
}

${ }^{1}$ Gazi University, Science \& Arts Faculty, Department of Biology, 06500 Ankara, Turkey. ozdikmen@gazi.edu.tr

\begin{abstract}
A new name for the Neotropical genus Aniarella Enderlein (Diptera, Sciaridae). A junior homonym was detected among neotropical sciarid flies genera and the following replacement name is proposed: Novaniarella nom. nov. for Aniarella Enderlein, 1911 (nec Bolivar, 1906). Accordingly, new combinations are herein proposed for the species currently included in this genus: Novaniarella azteca (Lane, 1959) comb. nov., Novaniarella brevis (Rubsaamen, 1894) comb. nov. and Novaniarella pelluscens
\end{abstract} (Enderlein, 1911) comb. nov.

KEYWORDS. Homonymy; new combinations; nomenclatural changes; replacement name; Sciaridae.

RESUMO. Um novo nome para o gênero neotropical Aniarella Enderlein (Diptera, Sciaridae). Um homônimo júnior foi descoberto entre os gêneros neotropicais de Sciaridae. O nome de substituição proposto é o seguinte: Novaniarella nom. nov. para Aniarella Enderlein, 1911 (nec Bolivar, 1906). As novas combinações necessárias são propostas aqui para as espécies atualmente incluídas nesse gênero: Novaniarella azteca (Lane, 1959) comb. nov., Novaniarella brevis (Rubsaamen, 1894) comb. nov. e Novaniarella pelluscens (Enderlein, 1911) comb. nov.

PALAVRAS-CHAVE. Homonímia; modificações nomenclaturais; nome de substituição; novas combinações; Sciaridae.

In an effort to eliminate homonymies among the genera of Neotropical Sciaridae, we found a sciarid genus which name had been used before in another insect order, resulting in a junior homonym. In accordance with the International Code of Zoological Nomenclature, we propose a replacement name for this genus.

\section{Novaniarella nom. nov.}

Aniarella Enderlein, 1911: 141 (Diptera, Nematocera, Bibionomorpha, Sciaridae). Preoccupied by Aniarella Bolivar, 1906: 394 (Orthoptera, Ensifera, Tettigonioidea, Tettigoniidae).

\section{Remarks on nomenclatural changes}

Enderlein (1911) described the Neotropical sciarid genus Aniarella, indicating as type species Aniarella pelluscens Enderlein, 1911, from Brazil. The name is still in use as a valid genus (e.g. Amorim, 1992). There is no generic synonyms under Aniarella. At present, this genus includes three species (including the type-species).

The generic name, however, was already preoccupied by Bolivar (1906), who proposed the objective replacement name Aniarella for a preoccupied genus name Aniara Brunner von Wattenwyl, 1878 in the family Tettigoniidae (Orthoptera), still used as a valid genus name and which also has no generic synonyms. This tettigoniid genus now includes five species (including the type-species): Aniarella ferracciui Piza, 1977; A. minor Bruner, 1915; A. proxima (Brunner von Wattenwyl, 1891); A. punctulata (Brunner von Wattenwyl, 1878) and $A$. typica (Brunner von Wattenwyl, 1878).
The generic name Aniarella Enderlein, 1911, hence, is a junior homonym of the generic name Aniarella Bolivar, 1906. According to Article 60 of the International Code of Zoological Nomenclature (ICZN 1999), we propose here the replacement name Novaniarella for Aniarella Enderlein, 1911.

\section{Catalogue for Novaniarella nom. nov.}

Novaniarella nom. nov. for Aniarella Enderlein, 1911 (nec Bolivar, 1906). Type-species, Aniarella pelluscens Enderlein, 1911.

Novaniarella azteca (Lane, 1959): 76, figs. 4 (male terminalia), 34 (wing) (as Neosciara). Type-locality: Mexico, Districto Federal, Xochimilco. Distr. - Mexico. Holotype male, Museu de Zoologia da Universidade de São Paulo (MZSP). Comb. nov.

Aniarella azteca (Lane, 1959); Amorim, 1992: 56 (catalogue).

Neosciara azteca Lane, 1959: 76 (original description).

Novaniarella brevis (Rubsaamen, 1894): 36, pl. 2, fig. 8 (wing), pl. 3, fig. 16 (male terminalia) (as Sciara). Type-locality: Colombia, Facatativa. Distr. - Colombia. Lectotype male, Zoologische Sammlungen, Museum für Naturkunde der Humbolt Universität, Berlin (BZM). Comb. nov.

Aniarella brevis (Rubsaamen, 1894): 36 (original description); Enderlein,

1911: 141 (new combination); Amorim, 1992: 56 (catalogue, lectotype designation).

Lycoria obscurans Enderlein, 1911: 161 (original description); Amorim, 1992: 56 (catalogue, synonymy).

Lycoriella pimpliformis Enderlein, 1911: 162 (original description); Amorim, 1992: 56 (catalogue, synonymy).

Sciara brevis Rubsaamen, 1894: 76 (original description); Enderlein, 1911: 141 (new combination); Amorim, 1992: 56 (catalogue).

Sciara nigrina Rubsaamen, 1894: 35 (original description); Enderlein, 1911: 141 (new combination); Amorim, 1992: 56 (catalogue, synonymy). 
Novaniarella pelluscens (Enderlein, 1911): 141 (as Aniarella). Type-locality: Brazil, Santa Catarina. Distr. - Brazil (Santa Catarina). Lectotype male, Museuo del Instituto de Zoología Agrícola, Maracay, Venezuela (IZV). Comb. nov.

Aniarella pelluscens Enderlein, 1911: 141 (original description); Amorim, 1992 (catalogue).

\section{REFERENCES}

Amorim, D. S. 1992. A catalogue of the family Sciaridae (Diptera) in the Americas South of the United States. Revista Brasileira de Entomologia 36: 5-77.

Bolivar, I. 1906. Rectificaciones y observaciones ortopterológicas. Boletin de la Real Sociedad Española de Historia Natural 6: 384-393.

Enderlein, G. 1911. Die phyletischen Beziehungen der Lycoriiden (Sciariden) zu den Fungivoriden (Mycetophiliden) und Itonididen (Cecidomyiiden) und ihre systematische Gliederung. Archive für Naturgeschichte 77 (Supplement 3): 116-201.

ICZN. 1999. International Code of Zoological Nomenclature. Fourth Edition. London, The International Trust for Zoological Nomenclature, $306 \mathrm{p}$.

Lane, J. 1959. On Neotropical Neosciara (Diptera, Sciaridae). Studia Entomologica 2: 69-104.

Rübsaamen, E. H. 1894. Die aussereuropäischen Trauermücken des Königl. Museums für Naturkunde zu Berlin. Berliner Entomologische Zeitschrift 39: 17-42.

Received 25/12/2009; accepted 14/1/2011

Editor: Silvio Shigueo Nihei 